\title{
Interaction of Antimicrobial Lipopeptides with Bacterial Lipid Bilayers
}

\author{
Ganesh Shahane ${ }^{1} \cdot$ Wei Ding $^{2} \cdot$ Michail Palaiokostas $^{2} \cdot$ Helena S. Azevedo $^{2} \cdot$ Mario Orsi $^{3}$ (I)
}

Received: 24 January 2019 / Accepted: 3 May 2019 / Published online: 16 May 2019

(c) The Author(s) 2019

\begin{abstract}
The resistance of pathogens to traditional antibiotics is currently a global issue of enormous concern. As the discovery and development of new antibiotics become increasingly challenging, synthetic antimicrobial lipopeptides (AMLPs) are now receiving renewed attention as a new class of antimicrobial agents. In contrast to traditional antibiotics, AMLPs act by physically disrupting the cell membrane (rather than targeting specific proteins), thus reducing the risk of inducing bacterial resistance. In this study, we use microsecond-timescale atomistic molecular dynamics simulations to quantify the interaction of a short AMLP (C16-KKK) with model bacterial lipid bilayers. In particular, we investigate how fundamental transmembrane properties change in relation to a range of lipopeptide concentrations. A number of structural, mechanical, and dynamical features are found to be significantly altered in a non-linear fashion. At $10 \mathrm{~mol} \%$ concentration, lipopeptides have a condensing effect on bacterial bilayers, characterized by a decrease in the area per lipid and an increase in the bilayer order. Higher AMLP concentrations of 25 and $40 \mathrm{~mol} \%$ destabilize the membrane by disrupting the bilayer core structure, inducing membrane thinning and water leakage. Important transmembrane properties such as the lateral pressure and dipole potential profiles are also affected. Potential implications on membrane function and associated proteins are discussed.
\end{abstract}

keywords Antimicrobial lipopeptides $\cdot$ Lipid bilayers $\cdot$ Molecular simulation $\cdot$ Molecular dynamics

\section{Introduction}

The emergence and spread of bacterial strains resistant to antibiotics represent a formidable global health problem. Alternative compounds such as antimicrobial peptides (AMPs) have therefore been developed to have broadspectrum activity while being less likely to trigger bacterial resistance (Koczulla and Bals 2003). Most AMPs are cationic in nature due to the large number of positively charged lysine and arginine residues, thus promoting attractive electrostatic interactions with anionic bacterial membranes

Electronic supplementary material The online version of this article (https://doi.org/10.1007/s00232-019-00068-3) contains supplementary material, which is available to authorized users.

Mario Orsi

Mario.Orsi@uwe.ac.uk

1 Institute of Bioengineering, Queen Mary University of London, Mile End Road, London E1 4NS, UK

2 School of Engineering \& Materials Science, Queen Mary University of London, Mile End Road, London E1 4NS, UK

3 Department of Applied Sciences, University of the West of England, Coldharbour Lane, Bristol BS16 1QY, UK (while membranes of mammalian cells are approximately neutral). AMPs are known to destabilize bilayers either by toroidal pore formation (Sengupta et al. 2008) or by a number of proposed models such as the carpet model (Shai and Oren (2001) or the barrel-stave model (Ehrenstein and Lecar 1977), leading to outflow of crucial nutrients and ions and eventually bacterial cell death. However, AMPs are not without drawbacks, and despite still holding promise, they have been shown to be susceptible to breakdown by peptidases when used in vivo (Hancock and Sahl 2006; Straus and Hancock 2006). Moreover, AMPs are far larger than usual drug-like molecules and thus more complex and expensive to synthesize.

To develop drugs that retain antimicrobial properties and circumvent drawbacks, Avrahami and Shai developed an approach to conjugate fatty acids to short non-active cationic peptides, providing the resulting molecules with antimicrobial properties while controlling their size (Avrahami and Shai 2004; 2003). Specifically, in pioneering studies by Shai and coworkers (Makovitzki et al. 2006; 2008), a set of di-, tri-, and tetrapeptides were attached to palmitoyl chains through their N-terminus, creating a new class of short cationic lipopeptides. Several of them revealed 
strong antibacterial and antifungal activities as monitored in vitro, without causing any significant hemolysis. Experiments were performed to measure macroscopic properties such as dye leakage from calcein-encapsulated small unilamellar vesicles (SUVs) via membrane rupture, measuring entrance of dyes and probes in ruptured cells, determination of cell growth inhibition from microplate autoreaders, and visualization of cell damage using transmission electron microscopy (TEM). Another subsequent study revealed the effectiveness of such lipopeptides for clearing infections in vivo (Vallon-Eberhard et al. 2008). The structural simplicity of these antimicrobial lipopeptides (AMLPs) makes them relatively easy to synthesize and analyze in relation to their antimicrobial mechanisms.

Though AMLPs have antibacterial and antifungal features, their mode of action has been revealed to be notably different from that of AMPs (Makovitzki et al. 2006, 2008; Horn et al. 2013; Sikorska et al. (2014; Grasso et al. 2018). A number of molecular dynamics (MD) simulation studies have shown that interactions with bacterial lipid bilayers generally follow a three-step process: initial selfassembly of AMLPs into micelles, binding and insertion of the micelle into the lipid bilayer, and subsequent scattering of the AMLPs throughout the bilayer (Horn et al. 2013; Sikorska et al. 2014). Particularly, the self-assembly and cluster formation processes with peptide sequences of varying lengths were meticulously characterized using coarsegrained (CG) models by Sikorska et al. (2014); a key finding from the study was that an increase in peptide sequence increases its critical micellar concentration (CMC) and decreases the aggregation number. While increased steric interactions in the AMLP headgroups were found to be responsible for an increase in $\mathrm{CMC}$, the reason for a decrease in aggregation number was intuitively ascribed to increased electrostatic repulsion between micelles preventing their fusion into larger ones. Though AMLPs are independently capable of spontaneous self-assembly, the studies also show that the process can occur concurrently in association with the bilayer surface (Sikorska et al. 2014). Binding of AMLP clusters is then accompanied by formation of 1-palmitoyl-2-oleoyl-sn-glycero-3-phosphoglycerol (POPG)-rich domains at the binding site, caused by preferential electrostatic interactions of negatively charged PG headgroups with cationic lysines (K) (Horn et al. 2013; Sikorska et al. 2014).

While it is clear that considerable work has been done with respect to analyzing self-assembly and binding of AMLPs to model bacterial bilayers, previous studies (as briefly reviewed above) typically lack systematic analyses of the effects of AMLPs concentration on key structural, dynamical, transmembrane, and elastic properties. To address this issue, in this study we use atomistic MD simulations to examine and quantify crucial properties of a simple model of gram-negative bacterial inner membrane bilayer for a range of AMLP concentrations. The bacterial bilayer composition is modeled as a mixture of POPE (1-palmitoyl-2-oleoyl-sn-glycero-3-phosphoethanolamine) and POPG (1-palmitoyl-2-oleoyl-sn-glycero-3-phosphoglycerol) lipids in a 2:1 ratio, as in previous work (Horn et al. 2012, 2013). The chosen AMLP consists of a 16-carbon aliphatic chain bound to three lysine residues at the $\mathrm{N}$-terminus (thus designated as $\mathrm{C} 16-\mathrm{KKK}$ ) and previous experimental research highlighted its potent antibacterial and antifungal activities (Makovitzki et al. 2008). By studying bilayers incorporating different lipopeptide concentrations, we aim at systematically quantifying the changes induced on key membrane physical properties. Specifically, we simulate a pure bacterial bilayer system, as well as three other systems comprising AMLPs at increasing concentrations of 10, 25, and $40 \mathrm{~mol} \%$. Fundamental structural properties such as area per lipid, bilayer thickness, electron density profiles, and deuterium-order parameters are investigated. Water permeation events and permeability coefficients are subsequently quantified over the microsecondslong trajectories. Furthermore, we calculate transmembrane lateral pressure and dipole potential profiles, two fundamental and yet often overlooked properties that play crucial roles in many membrane processes (Shearman et al. 2006; Wang 2012; Mouritsen 2005; Palaiokostas et al. 2018). The lateral pressure profile characterizes the inhomogeneous distribution of lateral stresses as a function of depth inside the bilayer. Given the nanoscopic thickness of lipid membranes, experimental determination of internal stresses is extremely difficult. Theory and simulation data predict the lateral pressure to be highly sensitive to depth, with magnitudes varying over several hundred bars across the membrane thickness (Cevc and Marsh 1987; Samuli and Vattulainen 2010). Significant forces therefore act on embedded proteins and permeating molecules (van den Brink-van der Laan 2004; Marsh 2007; Samuli and Vattulainen 2010). Moreover, the lateral pressure profile also underlies important elastic parameters, such as the bending rigidity modulus and spontaneous curvature, which determine the bilayer ability to bend and deform. As a consequence, the pressure profile is central to many membrane-related biological processes (Liu et al. 2006; McMahon and Gallop 2005; Chernomordik et al. 1995; Sergey and Bezrukova 2000; Cafiso 1998; Attard et al. 2000; Botelho et al. 2006; Orsi et al. 2011). The dipole (or electrostatic) potential arises from the preferential alignment of the dipole moments of water and lipid molecules; similar to the pressure profile, the dipole potential is also a transmembrane property of paramount biological relevance that is extremely difficult to study experimentally (Dreyer et al. 2013; Wang 2012). In this work, we quantify the effects of different concentrations 
of C16-KKK AMLP on the aforementioned properties of model bacterial bilayers, and discuss implications for antimicrobial activity and membrane function.

\section{Methods}

\section{System Preparation}

The main details of the lipid-lipopeptide bilayer systems under investigation are reported in Table 1. Four different bacterial bilayers with a 2:1 PE:PG lipid ratio were generated using Membrane Builder (Wu et al. 2014; Jo et al. 2009; 2007) from CHARMM-GUI (Jo et al. 2008). Of these, three systems were modified to incorporate the C16-KKK AMLPs (molecular structures are reported in Fig. 1). The AMLPs were created using Marvinsketch (2016) and were inserted throughout both bilayer leaflets by first concatenating the relevant coordinate files and then manually adjusting the AMLPs positions using the 'move' $\Rightarrow$ 'molecule' functions in VMD (Humphrey et al. 1996), thus producing three different bacterial bilayer systems with lipopeptide concentrations of 10,25 , and $40 \mathrm{~mol} \%$. The AMLPs locations were chosen to be approximately equidistant from one another within each leaflet. A minimization was conducted to remove any steric clashes between the lipids and lipopeptides using the steepest descent algorithm (Abraham et al. 2018). The bilayers were then solvated with the TIP3P water model as implemented in CHARMM36 (MacKerell et al. 1998; Klauda et al. 2010), followed by the addition of counterions to neutralize the overall charge. The systems were subjected to a final minimization step to ensure removal of any close contacts.

\section{Simulation Protocol}

All-atom simulations were performed using GROMACS version 5.1 (Van Der Spoel et al. 2005; Hess et al. 2008) and CHARMM36 force field (MacKerell et al. 1998; Klauda et al. 2010), under semi-isotropic NPT conditions ( $1 \mathrm{~atm}$ and $310 \mathrm{~K})$. Each system was equilibrated with the Berendsen

Table 1 Simulation systems

\begin{tabular}{llccl}
\hline System & $\mathrm{N}_{\text {lipids }}$ & $\mathrm{N}_{\text {lipopeptides }}$ & $\mathrm{N}_{\text {water }}$ & $\mathrm{N}_{\text {ions }}$ \\
\hline Bacterial & PE (88), PG (44) & 0 & 5940 & $44(+)$ \\
10\% AMLP & PE (88), PG (44) & 14 & 6141 & $2(+)$ \\
25\% AMLP & PE (100), PG (50) & 50 & 7520 & $100(-)$ \\
40\% AMLP & PE (100), PG (50) & 100 & 11850 & $250(-)$ \\
\hline
\end{tabular}

$\mathrm{N}_{\text {lipids }}$ is the total number of lipids, $\mathrm{N}_{\text {lipopeptides }}$ is the total number of C16-KKK lipopeptides, $\mathrm{N}_{\text {water }}$ is the total number of water molecules, and $\mathrm{N}_{\text {ions }}$ is the total number of ions in the system. Lipid names are abbreviated as PE (POPE) and PG (POPG) barostat (Berendsen et al. 1984) for $10 \mathrm{~ns}$, and was then simulated for further $2 \mu \mathrm{s}$ using the Parrinello-Rahman barostat (Parrinello and Rahman 1981) with a coupling time constant of 2 ps and (default) compressibility of $4.5 \cdot 10^{-5}$ bar $^{-1}$. The velocity rescale thermostat (Bussi et al. 2007) was used with a coupling time constant of $0.1 \mathrm{ps}$. A time step of $2 \mathrm{fs}$ was used for all simulations. The van der Waals and coulombic interactions were shifted to zero between 1 and $1.2 \mathrm{~nm}$ and $0-1.2 \mathrm{~nm}$, respectively. The Particle Mesh Ewald (PME) algorithm was used for computing long-range electrostatic interactions. A grid-based search procedure was used to update neighbor list every five steps. The SETTLE algorithm (Shuichi and Kollman 1992) was used to constrain bonds and angles in water molecules. All other hydrogenrelated bonds were constrained using the LINCS algorithm (Berk et al. 1997), with two iterations in every step for correcting rotational effects and a numerical expansion up to fourth order. Conventional periodic boundary conditions were applied in all three dimensions.

\section{Data Analysis}

We evaluated the following properties: area per lipid $\left(A_{\mathrm{L}}\right)$, bilayer thickness $\left(d_{\mathrm{HH}}\right)$, electron density profile $(\rho(z))$, fractional interactions, deuterium-order parameters $\left(S_{\mathrm{CD}}\right)$, water permeation, lateral diffusion coefficient $\left(D_{\mathrm{L}}\right)$, lateral pressure profile $(\Pi(z))$, dipole potential profile $(\Psi(z))$, bending modulus $\left(\kappa^{m}\right)$, and spontaneous curvature $\left(\mathrm{c}_{0}^{m}\right)$. Data analysis was performed over the last 251-2000 ns of each production run, while the first $250 \mathrm{~ns}$ were regarded as equilibration. Properties of interest were sampled every $250 \mathrm{ps}$, except for water permeation whereby a frequency of $50 \mathrm{ps}$ was instead used to accurately record permeation events. Averages and standard errors were estimated using seven trajectory blocks of $250 \mathrm{~ns}$ length each. Transmembrane properties were averaged over the two monolayers and symmetrized with respect to the bilayer center.

To estimate the average area per lipid $A_{\mathrm{L}}$, we used the freely available tool APL@VORO (Lukat et al. 2013).The phosphate atom in the lipid headgroup was used as 'key atom' to project coordinates on a plane and evaluate the relevant Voronoi diagrams. $A_{\mathrm{L}}$ values for POPE and POPG lipids were calculated separately in both monolayers and averaged to better determine the effects lipopeptides have on individual lipids. The electron density profiles were computed using the gmx density tool in GROMACS, with the simulation regions divided into 500 slabs along the $X Y$-plane. The bilayer thickness $d_{\mathrm{HH}}$ was computed as the head-to-head (P-P) distance between two peaks of the lipid phosphate electron density profiles. Order parameters were obtained using the $c a l c \_o p . t c l$ script which produces correct order parameters especially for the unsaturated lipid tails as demonstrated in a previous study (Piggot et al. 2017). Water 
permeation events were calculated using gmx select wherein the number of water oxygens with z-coordinate within the defined boundaries of the bilayer core is counted in every frame.

Normalized fractional interactions were computed for the three components POPE, POPG, and C16-KKK AMLP using a procedure previously employed to characterize the degree of lipid associations in complex membranes (Kolds $\varnothing$ et al. 2014) and phase formations (de Jong et al. 2013). In particular, the relative number of contacts of a component was calculated with respect to each of the other components. The total number of contacts within a distance of $6 \AA$ for each component was obtained using the gmx mindist tool in GROMACS and then corrected for the total number of molecules in the respective systems. The fractional interaction was then obtained as the ratio of number of contacts with a particular component to the total number of contacts formed with all components. For a two- and three-component system, a fraction of 0.5 and 0.33 , respectively, correlate to a randomly mixed bilayer. Such interactions are not necessarily symmetric since the clustering and density of specific components will, for example, make the number of contacts between lipid $\mathrm{X}$ and lipid $\mathrm{Y}$ different from lipid $\mathrm{Y}$ contacts with lipid $\mathrm{X}$ as a consequence of number of nearest neighbors.

Lipid lateral diffusion coefficients were obtained from the linear-fitted slope of averaged two-dimensional mean square displacement (MSD) from $g m x m s d$, with the initial reference point reset every $50 \mathrm{~ns}$. For the lateral pressure profile, a custom version of GROMACS, the GROMACS-LS package (Vanegas et al. 2014; Torres-Sánchez et al. 2015) was used to rerun trajectories and output local stress tensors in 3D. The long-range electrostatic solver is not utilized in GROMACS-LS and hence an increased cut-off distance of $2 \mathrm{~nm}$ was used for Coulomb interactions as suggested by the package developers (Vanegas et al. 2014). The transmembrane dipole potential was calculated using the gmx potential tool in GROMACS.

\section{Results}

\section{Structural Properties}

Calculated area per lipid $\left(A_{\mathrm{L}}\right)$ and bilayer thickness $\left(d_{\mathrm{HH}}\right)$ values for all the systems are shown in Fig. 2, with the numerical values reported in the supplementary information (Table S1). For the AMLP-free bacterial bilayer, both lipids exhibit $A_{\mathrm{L}}$ values in agreement with previous literature results from experimental (Rand et al. 1988; Kučerka et al. 2012) and simulation (Skjevik et al. 2015; Tolokh et al. 2009) studies. As shown in Fig. 2(left), both lipids display a similar trend in the presence of AMLPs. Specifically, the area per lipid $A_{\mathrm{L}}$ decreases upon addition of $10 \%$ AMLPs. However, it should be noted that the quantitative decrease is quite small, of $\sim 3 \%$. A gradual increase in AMLP concentration to 25 and $40 \%$ results in a corresponding increase in $A_{\mathrm{L}}$. Interestingly, by comparing the extreme cases of pure bacterial bilayer and 40\% AMLPs, for POPG lipids there is only a slight net reduction of $\sim 1.7 \%$ while for POPE lipids we can observe a net increment of $\sim 5 \%$ in $A_{\mathrm{L}}$ values (with respect to the pure, AMLP-free system).

The data for bilayer thickness $d_{\mathrm{HH}}$ are displayed in Fig. 2(right). It can be noted that $d_{\mathrm{HH}}$ does not change significantly upon the addition of $10 \%$ AMLP. However, further additions of lipopeptides at 25 and $40 \%$ concentration bring about significant decreases in $d_{\mathrm{HH}}$. In particular, the $d_{\mathrm{HH}}$ decrease reaches $\sim 17 \%$ for the difference between the pure and the $40 \%$ AMLP systems. This can be seen clearly in the electron densities calculated for the lipid phosphate atoms, reported in Fig. S2 of the supplementary material.

The electron density profiles $(\rho(z))$ for all simulated bilayers are shown in Fig. 3. Overall, the addition of lipopeptides is accompanied by variable changes in peak magnitudes and widths. The most significant effects are observed for the $40 \%$ AMLP concentration, whereby the profile becomes considerably smoother compared to the pure bilayer system, due to a decrease in the magnitude of the main peaks and central trough, as well as an increase in corresponding width. In particular, in the tails region, this effect can be interpreted as an increase in disorder brought about by the high concentration of AMLPs. The increase in electron densities in the bilayer tails core for the 25 and 40\% AMLP systems also indicates lipid chain interdigitation, which we further confirmed by visualization in VMD (Humphrey et al. 1996) (see simulation snapshots in Fig. S3 of the supplementary material). Interdigitation of lipid tails is also consistent with the observed reduction in membrane thickness.

\section{Order Parameters}

The acyl chain deuterium-order parameters $\left(S_{\mathrm{CD}}\right)$ were calculated to quantify the effect of changes in the lipopeptide concentration on the structure of the bilayer hydrophobic core:

$S_{\mathrm{CD}}=\frac{1}{2}\left\langle 3 \cos ^{2} \theta-1\right\rangle$,

where $\theta$ is the angle between the $\mathrm{C}-\mathrm{D}$ vector and the axis normal to the bilayer surface. In Fig. 4, we show the average order parameters for the acyl chain carbon atoms of $\mathrm{PE}$ and PG lipids in all simulated systems. The $S_{\mathrm{CD}}$ values for all sn-1 chains (Fig. 4a, c) exhibit similar qualitative characteristics, in that they decrease from the position of the glycerol carbon to the end of the chain, a trend usually seen 
in fully saturated hydrocarbon tails. The $s n$ - 2 chains (Fig. $4 \mathrm{~b}$, d) also all share similar qualitative features, with the typical trough midway along the chains corresponding to the double-bonded carbon atoms.

The presence of lipopeptides at $10 \%$ concentration marginally increases the overall order of the PE and PG lipid tails, an observation consistent with the study of a similar lipopeptide (C16-KGGK) done by Horn et al. (2013). However, at higher lipopeptide concentrations significant drops in order parameters are observed, especially for the $40 \%$ system. This sharp increase in tails disorder is expected to promote water leakage, which suggests a possible antimicrobial mode of action; this hypothesis is further investigated in the following section. Moreover, in typical (peptide free) bilayers it is common to observe an anticorrelation between lipid-order parameters and lipid area, i.e., lower areas usually correspond to higherorder parameters. This relation is disrupted by the presence of AMLPs in our simulations, as it can be seen by comparing lipid areas (Fig. 2) with order parameters (Fig. 4).

\section{Water Permeation}

To measure any water leakage effect induced by the observed change in order parameters, we investigated the permeation of water molecules into the hydrophobic region of all simulated membranes. We defined a region in the bilayer core comprising the volume within $3 \AA$ from the $z=0$ plane at the bilayer center (see also Fig. S4 in the supporting information). As in previous work (Hong et al. 2014), a permeation event is recorded when a water molecule enters this region. It should be noted that while this approach captures all permeation events across the membrane, it may also produce false positives, whereby a counted water molecule fails to fully penetrate across and instead bounces back into the water phase where it came from. Though this limitation will prevent direct comparisons with standard experimental permeation measurements, we are still able to compare permeation counts in relation to different AMLP concentrations.

Figure 5a shows the cumulative number of permeation events during the simulations conducted. In particular, we recorded a total of $91,71,154$, and 554 permeation events at the end of $2000 \mathrm{~ns}$ trajectory run in bacterial, 10, 25, and 40\% AMLP systems, respectively, corresponding to, on average, a permeation event every $\sim 22, \sim 28, \sim 13$, and $\sim 3.6 \mathrm{~ns}$, respectively. Compared to the bacterial bilayer, water permeation marginally decreases for the 10\% AMLP system, increases considerably for the 25\% AMLP system, and increases manifold for the 40\% AMLP system. We then calculated corresponding permeability coefficients $P_{\mathrm{W}}$ using a previously reported approach (Marrink et al. 2004; Orsi et al. 2008, 2010) based on Fick's law:

$P_{\mathrm{W}}=\frac{J_{\mathrm{W}}}{\Delta C_{\mathrm{W}} A}$, where $J_{\mathrm{W}}$ is the unidirectional flux of water (taken as half the total bidirectional permeation count) per total simulation time, $A$ is the interfacial area, and $\Delta C_{\mathrm{W}}$ is the water concentration gradient calculated as $\Delta C_{\mathrm{W}}=\mathrm{C}_{W}^{\text {water phase }}-\mathrm{C}_{W}^{\text {hydrocarbon core }}=30 \mathrm{~nm}^{-3}$, assuming $\mathrm{C}_{W}^{\text {hydrocarboncore }}=0 \mathrm{~nm}^{-3}$. The permeability coefficients as a function of AMLP concentration are depicted in Fig. 5b (numerical values can be found in the supplementary information Table S1). It can be seen that water permeation is considerably enhanced for the 40\% AMLP system. Such an effect is correlated with the previously observed decrease in order parameters and increase in lipid area at $40 \%$ AMLP concentration.

\section{Fractional Interactions}

To better understand the nature of lipid-lipid and lipid-AMLP interactions, we calculated fractional interactions between them in all the simulated systems; results are displayed in Fig. 6. In the bacterial bilayer, PE lipids interact favorably with both PG and other PE lipids with $\sim 41$ and $59 \%$ of total contacts, respectively (Fig. 6a). PG lipids however interact almost only with PE ( $\sim 64 \%$ of all contacts) and rarely with other PG lipids.

The preference of PG-PE interactions in bacterial bilayers (Murzyn et al. 2005) and the 'stabilizing effect' PG lipids have on PE lipids (Tari and Huang 1989) are considered as the main regulatory mechanisms evolved by bacteria to control membrane permeability. Such behavior of PG lipids could be intuitively expected due to the presence of a negative charge on its headgroup, causing increased electrostatic repulsion between neighboring PG lipids and more favorable interactions with PE lipids via hydrogen bonding. These observations are in reasonable agreement with a previous MD study on the interaction between PE and PG lipids in a model bacterial bilayer by Murzyn et al. (2005). Introducing AMLPs in bacterial bilayers however disrupts this equilibrium of PG-PE interactions. PG lipids across all concentrations show a significant preference for lipopeptides over $\mathrm{PE}$ and PG lipids. As such, decreased PG-PE interactions could destabilize a bacterial bilayer by making it more susceptible to structural defects and permeation by polar and lipophilic molecules (Tari and Huang 1989).

\section{Lateral Diffusion}

The lateral diffusion coefficient $\left(D_{\mathrm{L}}\right)$ describes the long-range motion of lipids along the $X Y$-bilayer plane. The $D_{\mathrm{L}}$ values obtained are presented in Table 2 . For PE and PG lipids, our values of $5.42 \pm 0.25$ and $5.01 \pm 0.25 \times 10^{-7} \mathrm{~cm}^{2} / \mathrm{s}$ for the bacterial system are about $\sim 50 \%$ higher than the ones previously reported (Hong et al. 2014; Murzyn et al. 2005) for 3:1 PE:PG systems 
Table 2 Lateral diffusion coefficients $\left(10^{-7} \mathrm{~cm}^{2} / \mathrm{s}\right)$

\begin{tabular}{lll}
\hline System & POPE & POPG \\
\hline Bacterial & $5.42 \pm 0.25$ & $5.01 \pm 0.25$ \\
$10 \%$ AMLP & $0.77 \pm 0.06$ & $0.85 \pm 0.08$ \\
$25 \%$ AMLP & $0.71 \pm 0.04$ & $0.75 \pm 0.06$ \\
$40 \%$ AMLP & $1.22 \pm 0.06$ & $1.32 \pm 0.08$ \\
\hline
\end{tabular}

using atomistic simulations. Lipid lateral diffusion has been shown to be highly sensitive to the choice of force fields and simulation conditions (Poger and Mark 2012), and hence we attribute the differences observed to the use of a different force field (Murzyn et al. 2005) and a lower temperature (Hong et al. 2014) in the previous studies. Considering the effect of the addition of lipopeptides, Table 2 shows a drastic reduction by about $\sim 85 \%$ in lipid lateral diffusion with lipopeptide concentrations at 10 and $25 \mathrm{~mol} \%$. At $40 \mathrm{~mol} \%$, a similarly substantial (although slightly less pronounced) reduction of $\sim 75 \%$ can be observed. Overall, it is evident that the presence of cationic lipopeptides markedly reduces the lipid lateral diffusion, even at lower $(10 \%)$ concentrations. As far as we are aware, no other results have been previously reported in literature on lateral diffusion of bacterial membrane lipids in the presence of cationic lipopeptides.

\section{Lateral Pressure Profile}

The lateral pressure profile $(\Pi(z))$ describes the non-uniform, depth-dependent distribution of lateral stresses across the bilayer normal. An assessment of $\Pi(z)$ can provide molecular-level insights into the interactions between lipids and other membrane constituents (such as proteins/peptides) and an estimate of the overall mechanical stability of the bilayer. Figure 7 depicts $\Pi(z)$ for all the simulated bilayer systems. The pure bacterial bilayer displays a trend which is characteristic of pure phospholipid bilayers in general (Cantor 1999; Orsi and Essex 2011; Ding et al. 2015). In particular, a large positive pressure peak of $\sim 340$ bar can be seen at the interface of the water and heads region at about $\sim 2.7 \mathrm{~nm}$ from the bilayer center. Such positive pressures indicate net repulsive forces as a result of steric and electrostatic interactions between the water molecules and lipid headgroups. Upon proceeding deeper into the bilayer, the pressure dips to a minimum at $\sim 1.8 \mathrm{~nm}$ forming sharp negative pressure troughs at the interface between the heads and tails region. Such negative pressures indicate net attractive forces of hydrophobic nature (Marsh 2007). The pressure profile in the bilayer center (tails region) is primarily characterized by three positive pressure peaks, that are postulated to arise from loss of entropy due to tight packing of lipid chains in the bilayer core (Mukhin and Baoukina 2005; Orsi et al. 2008).
Table 3 Elastic properties for all bilayer systems

\begin{tabular}{llr}
\hline System & $\kappa^{m}\left(10^{-20} \mathrm{~J}\right)$ & \multicolumn{1}{c}{$\mathrm{c}_{0}^{m}\left(\mathrm{~nm}^{-1}\right)$} \\
\hline Bacterial & $4.48 \pm 0.5$ & $0.009 \pm 0.005$ \\
$10 \%$ AMLP & $4.97 \pm 0.4$ & $-0.014 \pm 0.006$ \\
$25 \%$ AMLP & $4.32 \pm 0.9$ & $0.002 \pm 0.001$ \\
$40 \%$ AMLP & $1.55 \pm 0.1$ & $0.016 \pm 0.007$ \\
\hline
\end{tabular}

Upon addition of lipopeptides, significant changes in $\Pi(z)$ can be noted. Regarding the positive pressure peak at the interface of water and heads region, the magnitude decreases substantially for all systems, while the peak width increases remarkably for bilayers at 25 and $40 \%$ lipopeptide concentrations. Deeper in to the bilayer at the heads-tails interface, the large hydrophobic troughs are only slightly affected at $10 \%$ lipopeptide concentration (increase of $\sim 3.7 \%$ in attractive pressures), but undergo a noticeable change at $25 \%$ and a substantial one at $40 \%$; a major shift of the pressure trough occurs towards the bilayer core along with a decrease in pressure magnitudes of $\sim 150$ bar and $\sim 205$ bar at the two concentrations, respectively. Thus, with respect to the tails region, a gradual increase in lipopeptide concentration brings about a notable decrease in $\Pi(z)$, an effect which can be correlated with the decrease in order parameters noted earlier.

In general, it is apparent that the lateral pressure profiles for bacterial bilayer are affected in the presence of AMLPs. The changes are most pronounced for the 40\% AMLP system, an effect analogous to that observed previously for other properties. Additional quantitative analysis of the lateral pressure profiles based on elastic parameters is reported in the following section.

\section{Elastic Properties}

Fundamental elastic properties of lipid bilayers can be analytically related to the lateral pressure profile $\Pi(z)$, with the product of monolayer bending rigidity modulus $\left(\kappa^{m}\right)$ and monolayer spontaneous curvature $\left(\mathrm{c}_{0}^{m}\right)$ being equal to the first integral moment of $\Pi(z)$ :

$\kappa^{m} \mathrm{c}_{0}^{m}=\int_{0}^{l} z \Pi(z) d z$

where $z=l$ is in the water phase and $z=0$ is the bilayer core (Szleifer et al. 1990; Lipowsky and Sackmann 1995; Orsi and Essex 2013). With a standard numerical integration using the trapezoidal rule, the first integral moment of $\Pi(z)$ was obtained. Subsequently, $\kappa^{m}$ was evaluated using the empirical relation (Rawicz et al. 2000) $\kappa^{m}=k_{\mathrm{A}}\left(d_{\mathrm{HH}}-10\right)^{2} / 48$, with $d_{\mathrm{HH}}$ the bilayer thickness and 
Table 4 Summary of conclusions

\begin{tabular}{llllllllllll}
\hline System & Lipids & $A_{\mathrm{L}}$ & $d_{\mathrm{HH}}$ & $\rho(z)$ & $S_{\mathrm{CD}}$ & $P_{\mathrm{w}}$ & $D_{\mathrm{L}}$ & $\Pi(z)$ & $\kappa^{m}$ & $\mathrm{c}_{0}^{m}$ & $\Psi(z)$ \\
\hline Bacterial & $\mathrm{PE} / \mathrm{PG}$ & $=/=$ & $=$ & $=$ & $=$ & $=$ & $=/=$ & $=$ & $=$ & flat & $=$ \\
$10 \%$ AMLP & $\mathrm{PE} / \mathrm{PG}$ & $\downarrow / \downarrow$ & $=$ & $=$ & $\uparrow / \uparrow$ & $\downarrow$ & $\downarrow / \downarrow$ & $\downarrow$ (heads) & $=$ & $=$ & $\uparrow$ \\
$25 \%$ AMLP & $\mathrm{PE} / \mathrm{PG}$ & $\downarrow / \downarrow$ & $\downarrow$ & $\uparrow$ (tails) & $\downarrow / \downarrow$ & $\uparrow$ & $\downarrow / \downarrow$ & $\downarrow$ & $=$ & $=$ & $\uparrow$ \\
$40 \%$ AMLP & $\mathrm{PE} / \mathrm{PG}$ & $\uparrow / \downarrow$ & $\downarrow$ & $\uparrow($ tails) & $\downarrow / \downarrow$ & $\uparrow$ & $\downarrow / \downarrow$ & $\downarrow$ & & $=$ & $\downarrow$ (tails) \\
\hline
\end{tabular}

Conclusions derived in this study are summarized as above, wherein properties of the pure bacterial system are compared with systems containing AMLPs. Upward $(\uparrow)$ and downward $(\downarrow)$ arrows, respectively, correspond to increases and decreases in magnitudes of the said properties in comparison to those of the bacterial bilayer. Properties with two arrows (e.g., $\downarrow / \downarrow$ ) that are separated by a "/" correspond to an increase or a decrease in magnitudes of properties of PE / PG lipids in the said system in comparison to those of the bacterial bilayer. $P_{\mathrm{w}}$ is the water permeability coefficient as reported in Fig. $5 \mathrm{~b}$
$k_{\mathrm{A}}$ the area compressibility modulus (values reported in the supplementary information, Table S1). The spontaneous curvature $\mathrm{c}_{0}^{m}$ was then simply obtained as the ratio between the first integral moment of $\Pi(z)$ and the monolayer bending modulus $\kappa^{m}$. The values of the elastic properties for all simulated bilayers are reported in Table 3 .

The bending rigidity modulus $\kappa^{m}$ is arguably the single most important quantity in membrane biophysics (Watson et al. 2012) and determines the membrane's ability to resist bending in a number of biologically relevant processes such as membrane fusion (Liu et al. 2006), membrane trafficking of molecules (McMahon and Gallop 2005), and endocytosis (Chernomordik et al. 1995). As such, larger values of $\kappa^{m}$ correspond to greater rigidity of the bilayers. Our value of $\kappa^{m}$ for the 2:1 PE:PG bacterial bilayer is consistent with a previous one measured using a coarse-grained (CG) 3:1 PE:PG model (Fowler et al. 2016). Regarding the effect of lipopeptides, our results show an $11 \%$ increase in $\kappa^{m}$ with addition of $10 \%$ lipopeptide concentration. Interestingly, a further increase in lipopeptide concentration to $25 \%$ leads to a decrease of $\kappa^{m}$ down to $4.32 \cdot 10^{-20} \mathrm{~J}$, a value close to that of the pure bacterial bilayer. In the system with $40 \%$ lipopeptide concentration, $\kappa^{m}$ drops sharply to $1.55 \cdot 10^{-20} \mathrm{~J}$, a decrease of up to $\sim 65 \%$ as compared to the bacterial bilayer. Such a major drop can be correlated with the previously observed substantial decrease in magnitudes of the pressure profiles for the $40 \%$ AMLP system, as a reduction in internal stresses is expected to induce lower membrane rigidity.

In symmetrical bilayers, the monolayer spontaneous curvature $\mathrm{c}_{0}^{m}$ determines the intrinsic tendency of monolayers to curl if allowed to bend freely, rather than being constrained to a flat configuration as part of a lamellar bilayer structure (Lewis and Cafiso 1999). A positive $\mathrm{c}_{0}^{m}$ indicates a monolayer's tendency to bend towards the hydrocarbon chains and away from the aqueous phase (tendency to form micelles), while a negative $\mathrm{c}_{0}^{m}$ indicates the opposite tendency of bending towards the water phase (tendency to form inverse phases). In general, the more $\mathrm{c}_{0}^{m}$ deviates from 0 in a flat system, the more elastic energy is stored in the monolayer; beyond a certain threshold (depending on the system), increases in $\mathrm{c}_{0}^{m}$ may destabilize the flat structure. A small magnitude for $\mathrm{c}_{0}^{m}$ (close to zero), of either sign, indicates no significant tendency to curl, corresponding to a stable flat lamellar phase. In Table 3, it can be noted that $\mathrm{c}_{0}^{m}$ values for all systems are characterized by very low magnitudes, indicating high stability of the flat lamellar bilayer phases. As far as we know, this is the first study either in experiments or simulations to report $\mathrm{c}_{0}^{m}$ values for a model bacterial bilayer incorporating lipopeptides. Overall, our results show that the spontaneous curvature for a $2: 1 \mathrm{PE}: \mathrm{PG}$ bacterial bilayer is not significantly sensitive to the presence of the C16-KKK lipopeptides in it.

\section{Dipole Potential}

The dipole potential profiles $(\Psi(z))$ for all simulated systems are shown in Fig. 8. Profile decompositions highlighting the individual contributions of ions, water, and lipid/lipopeptide molecules can be found in the supplementary information (Fig. S7). As shown in Fig. 8, all profiles share similar qualitative features, regardless of the different lipopeptide concentrations. However, there are important concentrationdependent differences in the magnitudes of the potentials, whereby increasing amounts of C16-KKK induce increasingly larger values of $\Psi(z)$ across the water - heads interface. Beginning from a value of $0 \mathrm{~V}$ in the water phase, the potential rises rapidly across the lipid heads region forming local peaks at around $\sim 1.4 \mathrm{~nm}$ from the bilayer core, with values ranging from $\sim 0.5 \mathrm{~V}$ for the pure bacterial system to $\sim 0.6 \mathrm{~V}$ for the systems with 25 and $40 \%$ lipopeptides, with the lower $10 \%$ concentration displaying values in-between. The profiles then dip, forming local minima at $\sim 0.8 \mathrm{~nm}$ from the bilayer center, which roughly corresponds to the double bonds in the palmitoyl chains of lipids. In the membrane core, the dipole potential forms a global maximum for each of the individual profiles, with values of $\sim 0.71 \mathrm{~V}, \sim 0.74 \mathrm{~V}$, and $\sim 0.78 \mathrm{~V}$ for the bacterial, 10 , and $25 \%$ AMLP systems, respectively. Interestingly, the $40 \%$ AMLP system breaks the 
trend and forms the lowest global maximum at $\sim 0.68 \mathrm{~V}$. This can be related to the order parameters, which we observed to drop sharply for the $40 \%$ system; increased disorder in the tails region reduces dipolar alignment and ultimately leads to a decreased potential magnitude. As far as we are aware, there are no previous reports in literature on the dipole potential for bacterial lipid bilayers.
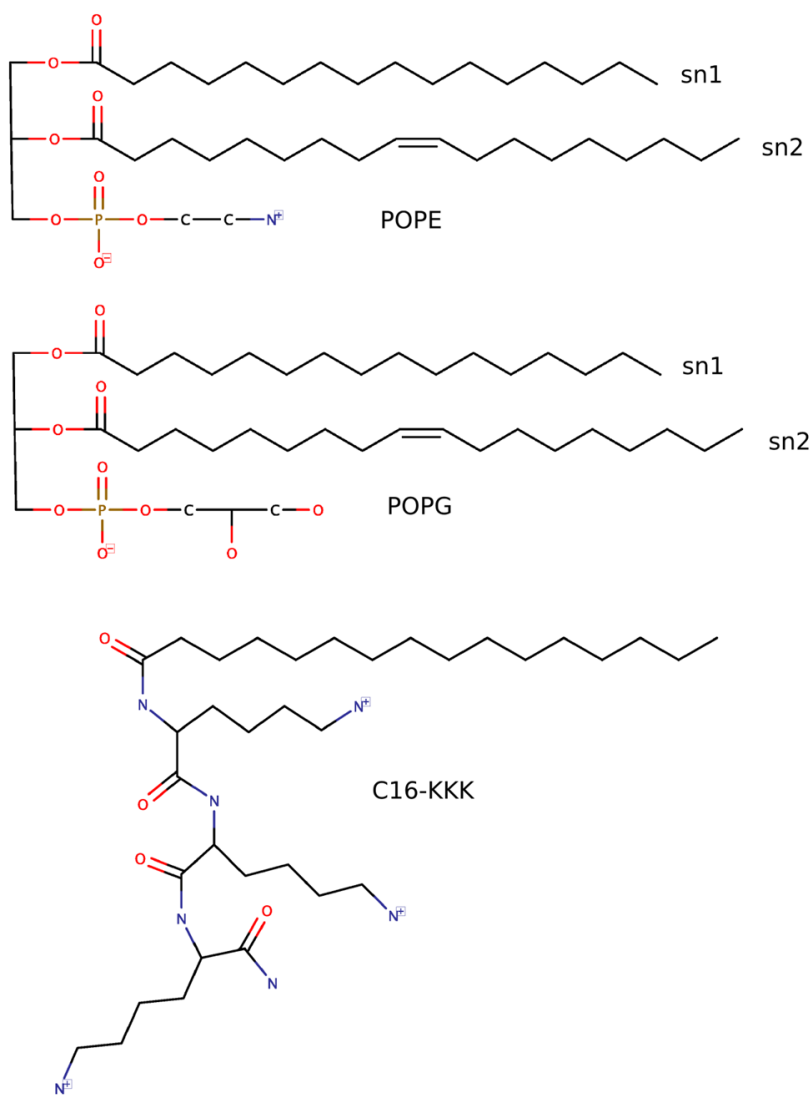

Fig. 1 Molecular structures of POPE (top), POPG (middle), and C16KKK (below). Hydrogens are not shown for clarity

\section{Discussion and Conclusion}

In recent times, there has been growing interest in developing short cationic antimicrobial lipopeptides (AMLPs) as a class of compounds that possess antimicrobial properties with fewer drawbacks compared to standard antimicrobial peptides (AMPs). While previous experimental studies have focused on quantifying bactericidal activities of various lipopeptides (Makovitzki et al. 2008, 2007, 2006), previous computational research focused on their self-assembly process and binding interactions with model bacterial bilayers (Horn et al. 2013; Sikorska et al. (2014). In this work, we studied mixed bilayer systems where the cationic C16KKK AMLPs are fully incorporated into a PE/PG bacterial bilayer. In particular, we deploy atomistic MD simulations to investigate the effect of C16-KKK AMLPs on a number of key physical properties of a model 2:1 PE:PG bacterial bilayer system, at concentrations of 10,25 , and $40 \mathrm{~mol} \%$. A concise summary of all conclusions is reported in Table 4 . With the exception of the monolayer spontaneous curvature, the properties investigated were shown to be notably affected by the cationic AMLPs, especially at the higher 25 and 40 mol\% concentration levels.

In particular, at 10\% AMLP concentration, the lipopeptides appear to have a condensing effect on bacterial bilayers, characterized by a significant decrease in the area per lipid. The AMLPs acyl chains are fully inserted in the bilayer core, while their lysines are largely hydrated and interact with both PG and PE headgroups. There is an overall increase in bilayer order, accompanied by a marked reduction in lateral diffusion for both lipids. While PG lipids preferentially interact with PEs in bacterial bilayers, in the 10\% AMLP system anionic PGs intuitively show preference for interaction with cationic lipopeptides while like-charge repulsions keep PG-PG and AMLP-AMLP interactions to a minimum. Increases in lipopeptide concentrations to 25 and $40 \%$ were observed to bring about significant disruption to the
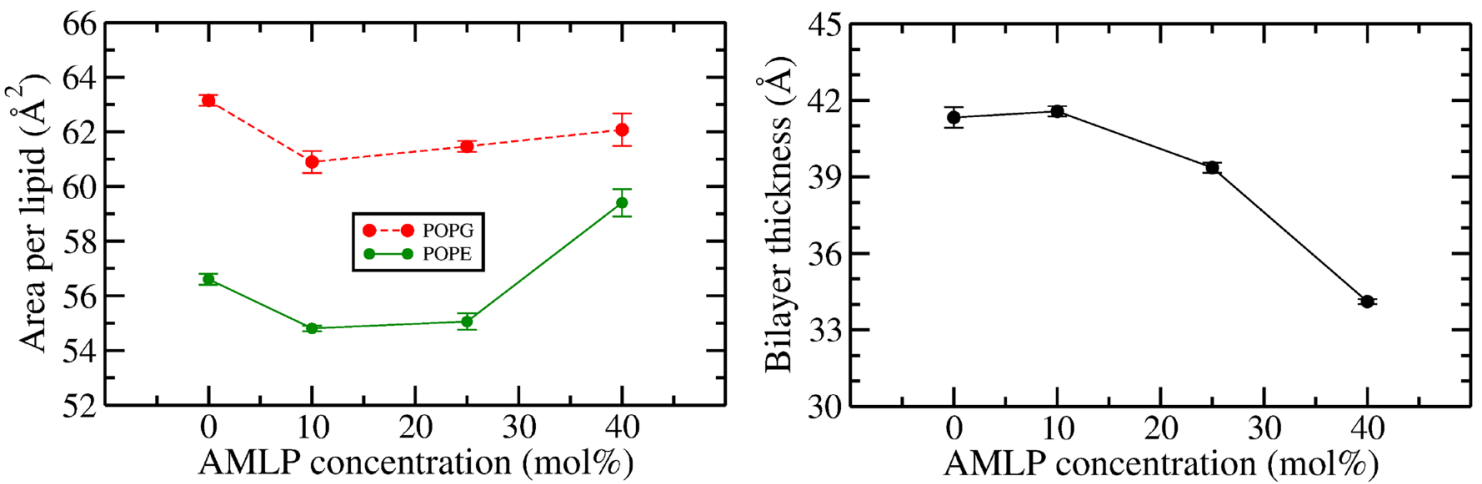

Fig. 2 Area per lipid (left) and bilayer thickness (right) values as a function of PA concentration. The area per lipid values were calculated for POPE and POPG lipids individually. Error bars represent standard errors 
bacterial lipid bilayer. The main effects involve an increase in lipid chain interdigitation, a decrease in order parameters and bending rigidity modulus, and an enhanced degree of spontaneous permeation of water molecules in the bilayer core, especially in the 40\% AMLP system. Thus, membrane stability is particularly impaired at the highest concentration

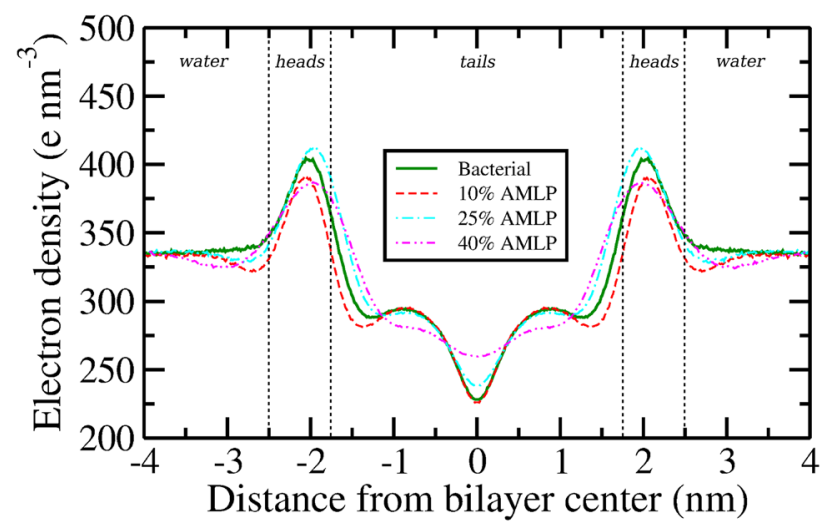

Fig. 3 Electron density profiles. Error bars, omitted here for clarity, are reported in the supplementary material

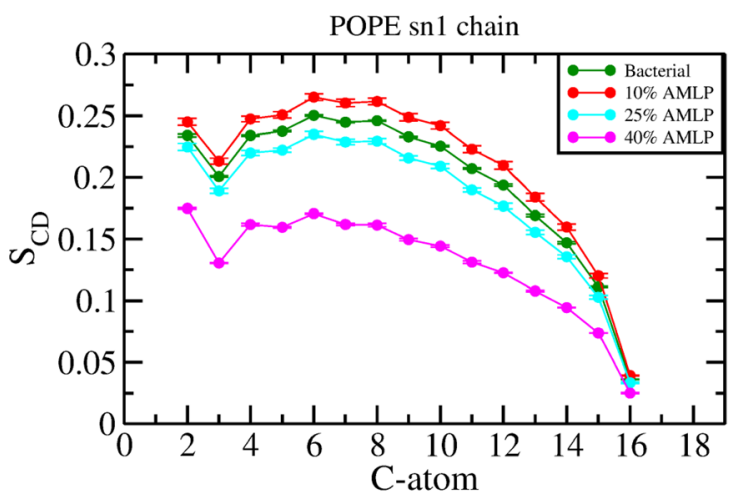

(a)

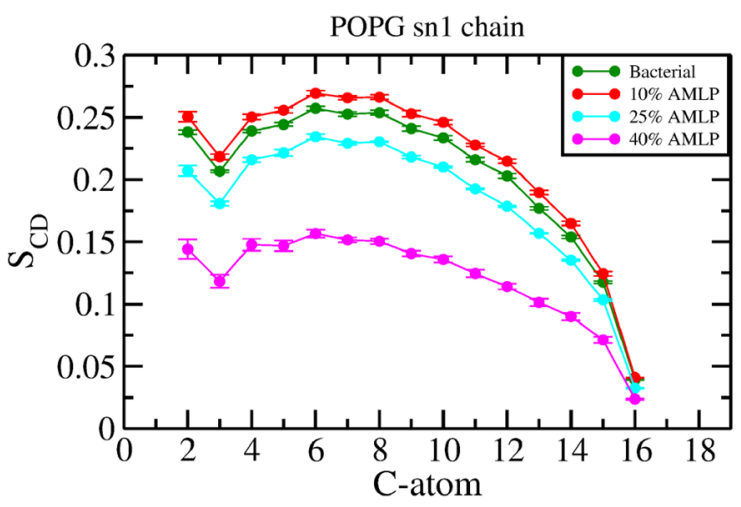

(c) of $40 \%$, which corresponds to the ratio 1 AMLP : 1 PE : 2 PG.

The lateral pressure profile is also substantially altered at $40 \%$ AMLP concentration, as we observed a systematic and substantial reduction in pressure magnitudes throughout the bilayer. Quantitatively, the pressure differences between the bacterial and 40\% AMLP systems are on the order of hundreds of bars, and changes of this size may play a role in membrane protein functionality (Cantor 1997). For example, the bacterial mechanosensitive channel (MS) is thought to play an important role in regulating turgor pressure around the cell (Perozo and Rees 2003), and the gating of these channels found in inner bacterial membranes of Escherichia coli is shown to be affected by changes in lateral pressure (Gullingsrud and Schulten 2004; Moe and Blount (2005). Previous studies have shown that, in response to a rapid osmotic shock, many of the cytoplasmic components such as solutes/water are ejected in to the surroundings by opening of the MS channels to relieve the osmotic imbalance (Perozo and Rees 2003). Thus a permanent leakage through the channel can lead to the death of bacteria, and an understanding of the relevant gating mechanism could lead to the development of new or

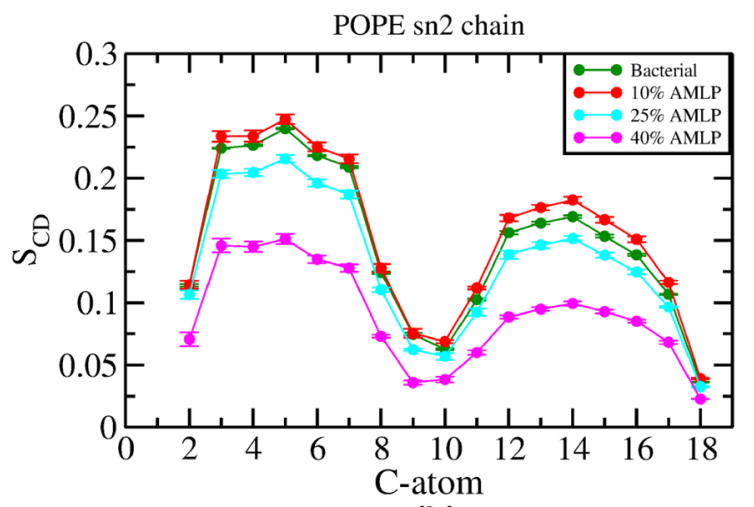

(b)

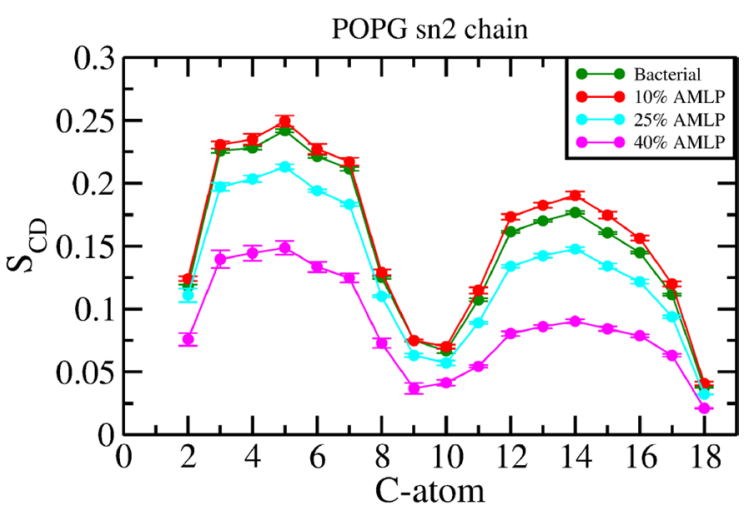

(d)

Fig. 4 Deuterium-order parameters $\left(\mathrm{S}_{\mathrm{CD}}\right)$ for a POPE sn-1 chain, b POPE sn-2 chain, $\mathbf{c}$ POPG sn-1 chain, and $\mathbf{d}$ POPG sn-2 chain. Dashes represent error bars 

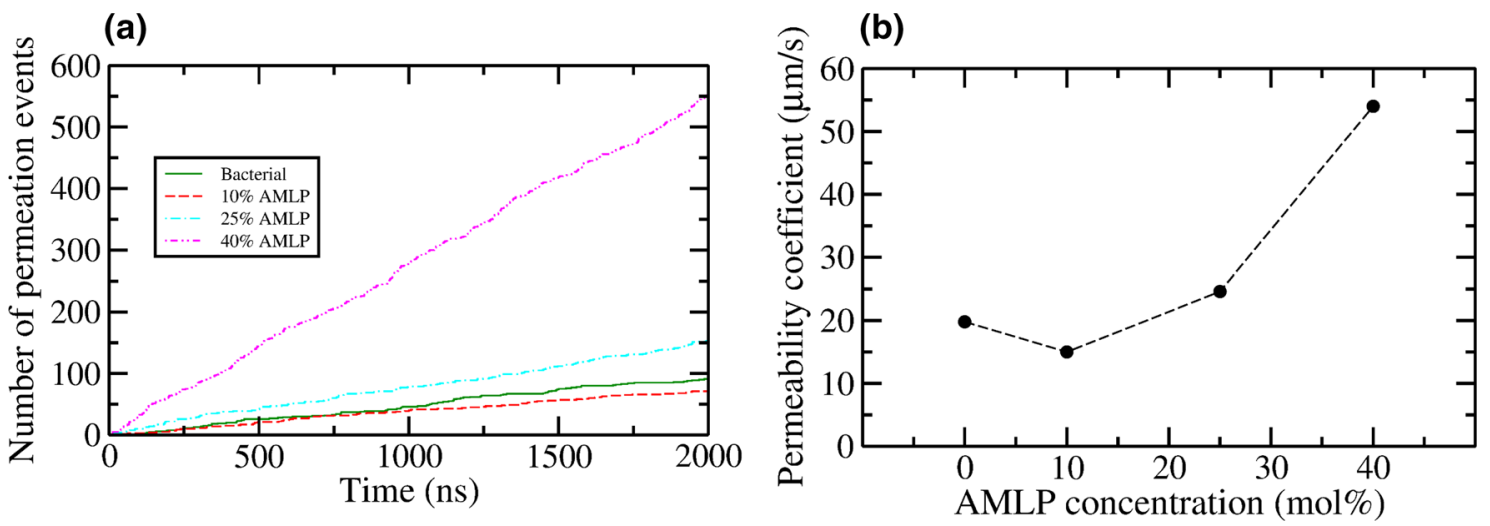

Fig. 5 Measurement of water permeation. a Cumulative number of water permeation events as a function of simulation time; $\mathbf{b}$ water permeability coefficient as a function of AMLP concentration

(a)

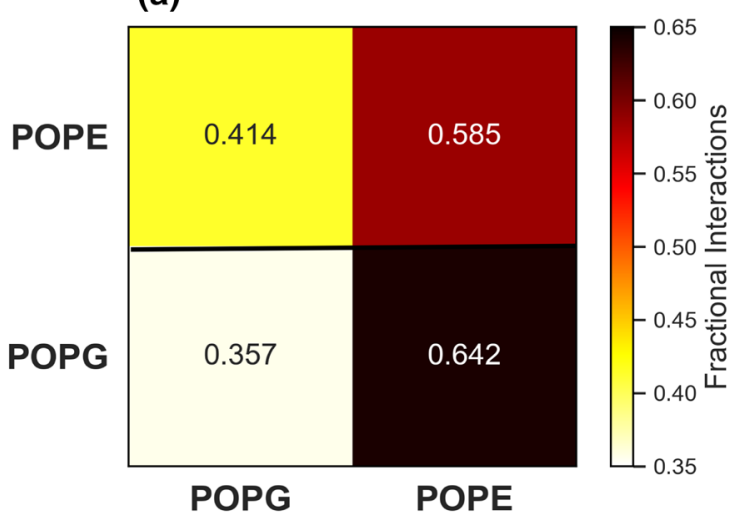

(c)

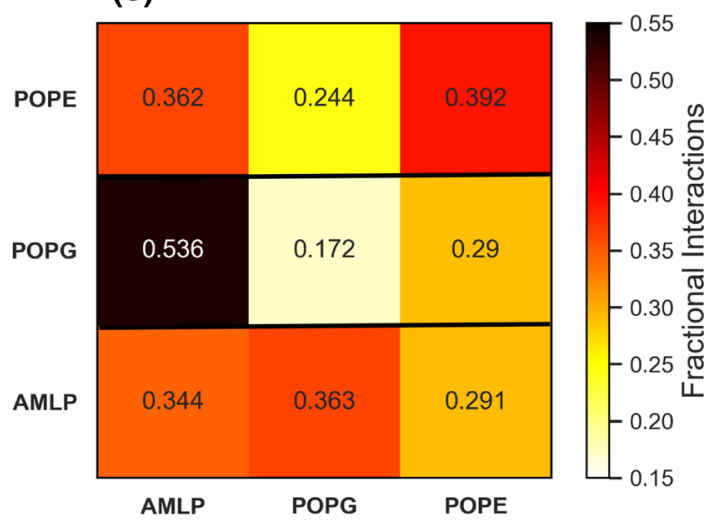

Fig. 6 Fractional interaction matrices for all the simulated bilayers. Each fractional interaction is the relative number of contacts between lipids as compared to all other contacts. Two lipids are defined as being in contact if the distance between their phosphate atoms is less than $6 \AA$. For AMLPs, the oxygen atom of the peptide bond connecting the first and the second lysine is considered for calculations. For
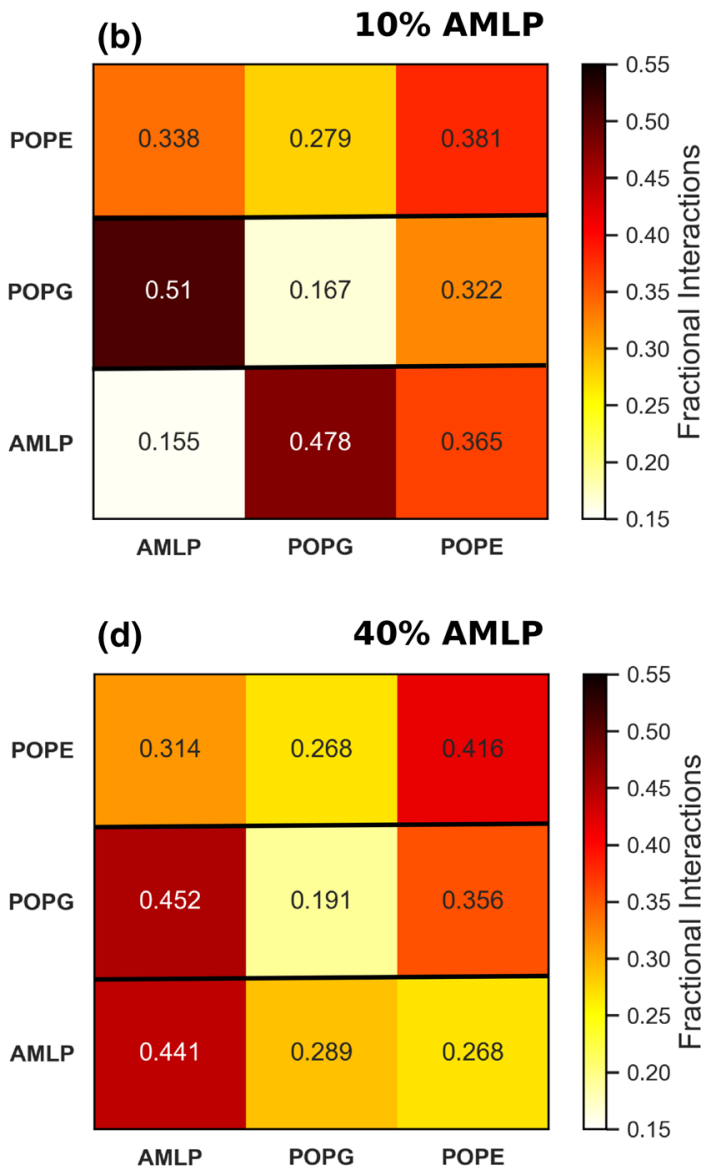

the bacterial bilayer, a fully random distribution of the two lipids would result in a fraction of 0.5 , while for the systems with AMLPs the fraction would be 0.33 . The fractions are arranged such that in each row the sum of all fractions for each component would be equal to 1 . This figure shows fractional interaction matrices of a bacterial bilayer, b 10\% AMLP, c 25\% AMLP, and d 40\% AMLP 


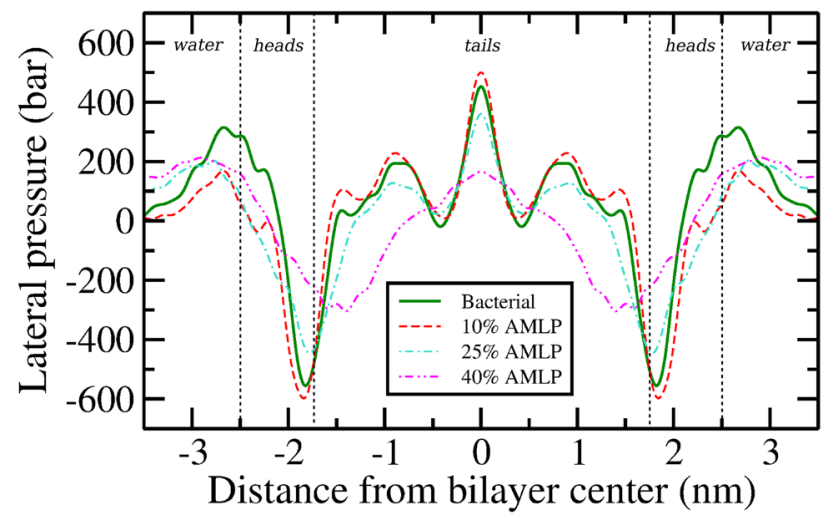

Fig. 7 Lateral pressure profiles. Error bars, omitted here for clarity, are reported in the supplementary material

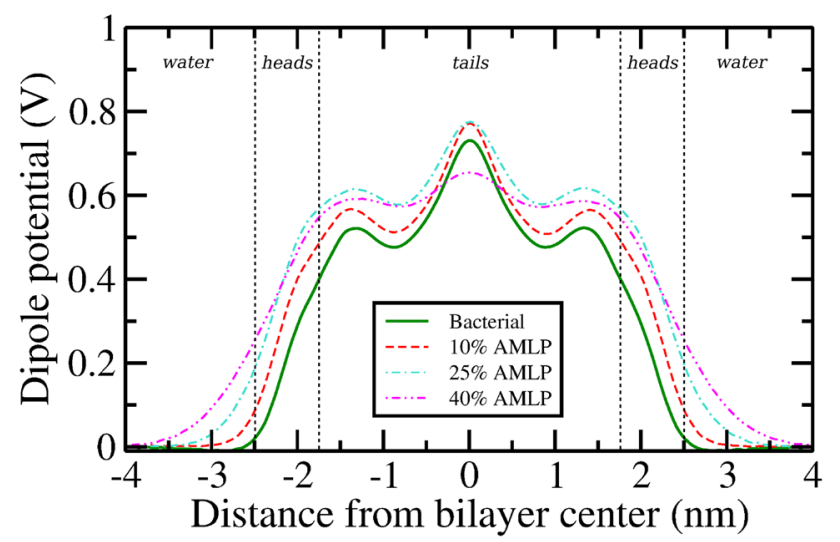

Fig. 8 Dipole potential profiles. Error bars, omitted here for clarity, are reported in the supplementary material

improved antimicrobial agents. Our results also suggest an antimicrobial mechanism of action whereby cationic lipopeptides cause increased rates of water permeation events in the bilayer core, as quantified by the increase in water permeation coefficients, especially for the $40 \mathrm{~mol} \%$ AMLP system. We speculate that the biological effects of AMLPs mainly arise from locally disordering the bilayer core and inducing leakage in inner bacterial membranes.

Overall, our results provide a quantitative molecular-level understanding of the experimental observations that cationic lipopeptides in general, and the C16-KKK AMLP in particular, are highly potent towards bacterial cells and operate by means of destabilizing their membranes (Makovitzki et al. 2008, 2007, 2006). Our work provides the basis for future simulations of more complex bacterial membranes, especially including mechanosensitive proteins, aimed at quantifying potential AMLP effects on protein conformational states.
Acknowledgements This project made use of time on the ARCHER supercomputer via the UK High-End Computing Consortium for Biomolecular Simulation (HECBioSim), supported by EPSRC (Grant No. EP/L000253/1). GS acknowledges financial support from the Institute of Bioengineering at Queen Mary University of London. HSA acknowledges the Seed Award in Science (Grant No. 210122/Z/18/Z) granted by the Welcome Trust.

\section{Compliance with Ethical Standards}

Conflict of interest The authors state that there are no conflicts of interests.

Human or Animal Subjects This article does not contain any studies with human or animal subjects.

Open Access This article is distributed under the terms of the Creative Commons Attribution 4.0 International License (http://creativeco mmons.org/licenses/by/4.0/), which permits unrestricted use, distribution, and reproduction in any medium, provided you give appropriate credit to the original author(s) and the source, provide a link to the Creative Commons license, and indicate if changes were made.

\section{References}

Abraham M et al (2018) Gromacs user manual 5.0.4

Attard GS, Templer RH, Smith WS, Hunt AN, Jackowski S (2000) Modulation of CTP:phosphocholine cytidylyltransferase by membrane curvature elastic stress. Proc Natl Acad Sci USA 97(16):9032-9036

Avrahami D, Shai Y (2003) Bestowing antifungal and antibacterial activities by lipophilic acid conjugation to D, L-amino acidcontaining antimicrobial peptides: a plausible mode of action. Biochemistry 42(50):14946-14956

Avrahami D, Shai Y (2004) A new group of antifungal and antibacterial lipopeptides derived from non-membrane active peptides conjugated to palmitic acid. J Biol Chem 279(13):12277-12285

Berendsen HJC, Postma JPM, van Gunsteren WF, DiNola A, Haak JR (1984) Molecular dynamics with coupling to an external bath. J Chem Phys 81(8):3684-3690

Berk H, Henk B, Herman BJC, Johannes FGEM (1997) Lincs: a linear constraint solver for molecular simulations. J Comput Chem 18(12):1463-1472

Botelho A, Huber T, Sakmar TP, Brown MF (2006) Curvature and hydrophobic forces drive oligomerization and modulate activity of rhodopsin in membranes. Biophys J 91(12):4464-4477

Bussi G, Donadio D, Parrinello M (2007) Canonical sampling through velocity rescaling. J Chem Phys 126(1):014101

Cafiso DS (1998) Dipole potentials and spontaneous curvature: membrane properties that could mediate anesthesia. Toxicol Lett 100-101:431-439

Cantor RS (1997) Lateral pressure in cell membranes: a mechanism for modulation of protein function. J Phys Chem 101(96):1723-1725

Cantor RS (1999) The influence of membrane lateral pressures on simple geometric models of protein conformational equilibria. Chem Phys Lipids 101(1):45-56

Cevc G, Marsh D (1987) Phospholipid bilayers: physical principles and models. Cell biology. Wiley, New York

Chernomordik L, Kozlov MM, Zimmerberg J (1995) Lipids in biological membrane fusion. J Membr Biol 146(1):1-14 
de Jong DH, Lopez CA, Marrink SJ (2013) Molecular view on protein sorting into liquid-ordered membrane domains mediated by gangliosides and lipid anchors. Faraday Discuss 161:347-363

Ding W, Palaiokostas M, Wang W, Orsi M (2015) Effects of lipid composition on bilayer membranes quantified by all-atom molecular dynamics. J Phys Chem B 119:15263-15274

Dreyer J, Zhang C, Ippoliti E, Carloni P (2013) Role of the membrane dipole potential for proton transport in gramicidin a embedded in a DMPC bilayer. J Chem Theory Comput 9(8):3826-3831

Ehrenstein G, Lecar H (1977) Electrically gated ionic channels in lipid bilayers. Q Rev Biophys 10(1):1-34

Fowler PW, Hélie J, Duncan A, Chavent M, Kolds $\varnothing$ H, Sansom MSP (2016) Membrane stiffness is modified by integral membrane proteins. Soft Matter 12(37):7792-7803

Grasso G, Muscat S, Rebella M, Morbiducci U, Audenino A, Danani A, Deriu MA (2018) Cell penetrating peptide modulation of membrane biomechanics by molecular dynamics. J Biomech 73:137-144

Gullingsrud J, Schulten K (2004) Lipid bilayer pressure profiles and mechanosensitive channel gating. Biophys J 86(6):3496-3509

Hancock REW, Sahl H-G (2006) Antimicrobial and host-defense peptides as new anti-infective therapeutic strategies. Nat Biotechnol 24:1551EP

Hess B, Kutzner C, van der Spoel D, Lindahl E (2008) GROMACS 4: Algorithms for highly efficient, load-balanced, and scalable molecular simulation. J Chem Theory Comput 4(3):435-447

Hong C, Tieleman DP, Wang Y (2014) Microsecond molecular dynamics simulations of lipid mixing. Langmuir 30(40):11993-12001

Horn JN, Romo TD, Grossfield A (2013) Simulating the mechanism of antimicrobial lipopeptides with all-atom molecular dynamics. Biochemistry 52(33):5604-5610

Horn JN, Sengillo JD, Lin D, Romo TD, Grossfield A (2012) Characterization of a potent antimicrobial lipopeptide via coarsegrained molecular dynamics. Biochim Biophys Acta-Biomembr 1818(2):212-218

Humphrey W, Dalke A, Schulten K (1996) VMD: visual molecular dynamics. J Mol Graph 14(1):33-38 27-28 February, 1996

Jo S, Kim T, Im W (2007) Automated builder and database of protein/ membrane complexes for molecular dynamics simulations. PLoS ONE 2(9):e880

Jo S, Kim T, Iyer VG, Im W (2008) CHARMM-GUI: a web-based graphical user interface for CHARMM. J Comput Chem 29(11):1859-1865

Jo S, Lim JB, Klauda JB, Im W (2009) CHARMM-GUI membrane builder for mixed bilayers and its application to yeast membranes. Biophys J 97(1):50-58

Klauda JB, Venable RM, Freites JA, O'Connor JW, Tobias DJ, Mondragon-Ramirez C, Vorobyov I, MacKerell AD Jr, Pastor RW (2010) Update of the CHARMM all-atom additive force field for lipids: validation on six lipid types. J Phys Chem B 114(23):7830-7843

Koczulla AR, Bals R (2003) Antimicrobial peptides: current status and therapeutic potential. Drugs 63(4):389-406

Kolds $\emptyset$ H, Shorthouse D, Hélie J, Sansom MSP (2014) Lipid clustering correlates with membrane curvature as revealed by molecular simulations of complex lipid bilayers. PLoS Comput Biol 10(10):e1003911

Kučerka N, Holland BW, Gray CG, Tomberli B, Katsaras J (2012) Scattering density profile model of POPG bilayers as determined by molecular dynamics simulations and amall-angle neutron and X-ray scattering experiments. J Phys Chem B 116(1):232-239

Lewis JR, Cafiso DS (1999) Correlation between the free energy of a channel-forming voltage-gated peptide and the spontaneous curvature of bilayer lipids. Biochemistry 38(18):5932-5938
Lipowsky R, Sackmann E (1995) Structure and dynamics of membranes. Number v. 1, pt. 1 in handbook of biological physics. Elsevier Science, New York

Liu J, Kaksonen M, Drubin DG, Oster G (2006) Endocytic vesicle scission by lipid phase boundary forces. Proc Natl Acad Sci USA 103(27):10277-10282

Lukat G, Krüger J, Sommer B (2013) APL@ Voro: a voronoi-based membrane analysis tool for GROMACS trajectories. J Chem Inf Model 53(11):2908-2925

MacKerell AD Jr, Bashford D, Bellott M, Dunbrack RL Jr, Evanseck JD, Field MJ, Fischer S, Gao J, Guo H, Ha S, Joseph-McCarthy D, Kuchnir L, Kuczera K, Lau FTK, Mattos C, Michnick S, Ngo T, Nguyen DT, Prodhom B, Reiher WE, Roux B, Schlenkrich M, Smith JC, Stote R, Straub J, Watanabe M, Wiórkiewicz-Kuczera J, Yin D, Karplus M (1998) All-Atom empirical potential for molecular modeling and dynamics studies of proteins. J Phys Chem B 102(18):3586-3616

Makovitzki A, Avrahami D, Shai Y (2006) Ultrashort antibacterial and antifungal lipopeptides. Proc Natl Acad Sci USA 103(43):15997-16002

Makovitzki A, Baram J, Shai Y (2008) Antimicrobial lipopolypeptides composed of palmitoyl di- and tricationic peptides: In vitro and in vivo activities, self-assembly to nanostructures, and a plausible mode of action. Biochemistry 47(40):10630-10636

Makovitzki A, Viterbo A, Brotman Y, Chet I, Shai Y (2007) Inhibition of fungal and bacterial plant pathogens in vitro and in planta with ultrashort cationic lipopeptides. Appl Environ Microbiol 73(20):6629-6636

Marrink SJ, de Vries AH, Mark AE (2004) Coarse grained model for semiquantitative lipid simulations. J Phys Chem B 108(2):750-760

Marsh D (2007) Lateral pressure profile, spontaneous curvature frustration, and the incorporation and conformation of proteins in membranes. Biophys J 93(11):3884-3899

Marsh D (2007) Lateral pressure profile, spontaneous curvature frustration, and the incorporation and conformation of proteins in membranes. Biophys J 93(11):3884-3899

Marvinsketch (version 17.2.27), calculation module developed by chemaxon. https://chemaxon.com/products/marvin. Accessed 30 September, 2016

McMahon HT, Gallop JL (2005) Membrane curvature and mechanisms of dynamic cell membrane remodelling. Nature 438:590 EP

Miyamoto S, Kollman PA (1992) An analytical version of the shake and rattle algorithm for rigid water models. J Comput Chem 13(8):952-962

Moe P, Blount P (2005) Assessment of potential stimuli for mechano-dependent gating of MscL: effects of pressure, tension, and lipid headgroups. Biochemistry 44(36):12239-12244

Mouritsen OG (2005) Life-as a matter of fat. The emerging science of lipidomics, 1st edn. Springer, Berlin

Mukhin SI, Baoukina S (2005) Analytical derivation of thermodynamic characteristics of lipid bilayer from a flexible string model. Phys Rev E 71(6):061918

Murzyn K, Róg T, Pasenkiewicz-Gierula M (2005) Phosphatidylethanolamine-phosphatidylglycerol bilayer as a model of the inner bacterial membrane. Biophys J 88(2):1091-1103

Orsi M, Essex JW (2011) The elba force field for coarse-grain modeling of lipid membranes. PLoS ONE 6(12):1-22

Orsi M, Essex JW (2013) Physical properties of mixed bilayers containing lamellar and nonlamellar lipids: insights from coarse-grain molecular dynamics simulations. Faraday Discuss 161:249-72 discussion 273-303

Orsi M, Haubertin DY, Sanderson WE, Essex JW (2008) A quantitative coarse-grain model for lipid bilayers. J Phys Chem B 112(3):802-815 
Orsi M, Michel J, Essex JW (2010) Coarse-grain modelling of DMPC and DOPC lipid bilayers. J Phys Condens Matter 22(15):155106

Orsi M, Noro MG, Essex JW (2011) Dual-resolution molecular dynamics simulation of antimicrobials in biomembranes. J R Soc Interface 8(59):826-841

Palaiokostas M, Ding W, Shahane G, Orsi M (2018) Effects of lipid composition on membrane permeation. Soft Matter 14:8496-8508

Parrinello M, Rahman A (1981) Polymorphic transitions in single crystals: a new molecular dynamics method. J Appl Phys 52(12):7182-7190

Perozo E, Rees DC (2003) Structure and mechanism in prokaryotic mechanosensitive channels. Curr Opin Struct Biol 13(4):432-442

Piggot TJ, Allison JR, Sessions RB, Essex JW (2017) On the calculation of acyl chain order parameters from lipid simulations. J Chem Theory Comput 13(11):5683-5696

Poger D, Mark AE (2012) J Chem Theory Comput. Lipid bilayers: the effect of force field on ordering and dynamics 8(11):4807-4817

Rand RP, Fuller N, Parsegian VA, Rau DC (1988) Variation in hydration forces between neutral phospholipid bilayers: evidence for hydration attraction. Biochemistry 27(20):7711-7722

Rawicz W, Olbrich KC, McIntosh T, Needham D, Evans E (2000) Effect of chain length and unsaturation on elasticity of lipid bilayers. Biophys J 79(1):328-339

Samuli Ollila OH, Ilpo V (2010) Chapter 2 lateral pressure profiles in lipid membranes: dependence on molecular composition. In: Mark SP (ed) Molecular simulations and biomembranes: from biophysics to function. The Royal Society of Chemistry, London, pp 26-55

Sengupta D, Leontiadou H, Mark AE, Marrink SJ (2008) Toroidal pores formed by antimicrobial peptides show significant disorder. Biochim Biophys Acta-Biomembr 1778(10):2308-2317

Sergey M, Bezrukova b (2000) Functional consequences of lipid packing stress. ELS EVI E R Curr Opin Colloid Interface Sci 5:237-243

Shai Y, Oren Z (2001) From "carpet" mechanism to de-novo designed diastereomeric cell-selective antimicrobial peptides. Peptides 22(10):1629-1641 Bacterial and anti bacterial peptides

Shearman GC, Ces O, Templer RH, Seddon JM (2006) Inverse lyotropic phases of lipids and membrane curvature. J Phys Condens Matter 18(28):S1105-S1124

Sikorska E, Dawgul M, Greber K, Iłowska E, Pogorzelska A, Kamysz W (2014) Self-assembly and interactions of short antimicrobial cationic lipopeptides with membrane lipids: ITC, FTIR and molecular dynamics studies. Biochim Biophys Acta 1838(10):2625-2634

Skjevik Å, Madej BD, Dickson CJ, Teigen K, Walker RC, Gould IR (2015) All-atom lipid bilayer self-assembly with the AMBER and CHARMM lipid force fields. Chem Commun 51(21):4402-4405
Straus SK, Hancock REW (2006) Mode of action of the new antibiotic for gram-positive pathogens daptomycin: comparison with cationic antimicrobial peptides and lipopeptides. Biochim Biophys Acta 1758(9):1215-1223

Szleifer I, Kramer D, Ben-Shaul A, Gelbart WM, Safran SA (1990) Molecular theory of curvature elasticity in surfactant films. J Chem Phys 92(11):6800-6817

Tari A, Huang L (1989) Structure and function relationship of phosphatidylglycerol in the stabilization of phosphatidylethanolamine bilayer. Biochemistry 28(19):7708-7712

Tolokh IS, Vivcharuk V, Tomberli B, Gray CG (2009) Binding free energy and counterion release for adsorption of the antimicrobial peptide lactoferricin B on a POPG membrane. Phys Rev E 80(3):031911

Torres-Sánchez 1, Vanegas JM, Arroyo M (2015) Examining the mechanical equilibrium of microscopic stresses in molecular simulations. Phys Rev Lett 114(25):258102

Vallon-Eberhard A, Makovitzki A, Beauvais A, Latgé J-P, Jung S, Shai Y (2008) Efficient clearance of aspergillus fumigatus in murine lungs by an ultrashort antimicrobial lipopeptide, palmitoyl-lysala-DAla-lys. Antimicrob Agents Chemother 52(9):3118-3126

van den Brink-van der Laan E, Killian JA, de Kruijff B (2004) Nonbilayer lipids affect peripheral and integral membrane proteins via changes in the lateral pressure profile. Biochim Biophys Acta 1666(1-2):275-288

Van Der Spoel D, Lindahl E, Hess B, Groenhof G, Mark AE, Berendsen HJC (2005) GROMACS: fast, flexible, and free. J Comput Chem 26(16):1701-1718

Vanegas JM, Torres-Sánchez A, Arroyo M (2014) Importance of force decomposition for local stress calculations in biomembrane molecular simulations. J Chem Theory Comput 10(2):691-702

Wang L (2012) Measurements and implications of the membrane dipole potential. Annu Rev Biochem 81:615-635

Watson MC, Brandt EG, Welch PM, Brown FLH (2012) Determining biomembrane bending rigidities from simulations of modest size. Phys Rev Lett 109(2):028102

Wu EL, Cheng X, Jo S, Rui H, Song KC, Dávila-Contreras EM, Qi Y, Lee J, Monje-Galvan V, Venable RM, Klauda JB, Im W (2014) CHARMM-GUI membrane builder toward realistic biological membrane simulations. J Comput Chem 35(27):1997-2004

Publisher's Note Springer Nature remains neutral with regard to jurisdictional claims in published maps and institutional affiliations. 